\title{
Analogs of solid nanoparticles as precursors of aromatic hydrocarbons
}

\author{
K. A. K. Gadallah ${ }^{1,2}$, H. Mutschke ${ }^{1}$, and C. Jäger ${ }^{3}$ \\ 1 Astrophysical Institute and University Observatory, Friedrich Schiller University, Schillergässchen 2/3, 07745 Jena, Germany \\ e-mail: Kamel.Gadallah71@gmail.com \\ 2 Astronomy Dpt., Faculty of Science, Al-Azhar University, Nasr City, PO Box 11884 Cairo, Egypt \\ ${ }^{3}$ Institute of Solid State Physics, Friedrich Schiller University of Jena, Helmholtzweg 3, 07743 Jena, Germany
}

Received 12 December 2012 / Accepted 20 April 2013

\begin{abstract}
Context. Aromatic $=\mathrm{CH}$ and $\mathrm{C}=\mathrm{C}$ vibrational bands have been observed within shocked interstellar regions, indicating the presence of aromatic emission carriers such as PAHs, which may have been created from adjacent molecular cloud material by interaction with a shock front.

Aims. We investigate the evolution of the aromatic $=\mathrm{CH}$ and $\mathrm{C}=\mathrm{C}$ vibrational modes at 3.3 and $6.2 \mu$ m wavelength in heated $\mathrm{HAC}$ materials, PAHs and mixed PAHs and HACs, respectively, aiming at an explanation of the evolution of carbonaceous dust grains in the shocked regions.

Methods. Materials used in these analogs (HAC and PAH materials) were prepared by the laser ablation and the laser pyrolysis methods, respectively. The transmission electron microscopy (TEM) in high-resolution mode was used as an analytical technique to characterize the aromatic layers in HACs. Spectroscopic analysis was prformed in the mid-IR range.

Results. A remarkable destruction of aliphatic structures in HACs has been observed with the thermal processing, while aromatic structures become dominating by increasing the diameters of the graphene layers. The aromatic bands at 3.3 and $6.2 \mu \mathrm{m}$, observed in the laboratory spectra of PAHs and of the combination of the PAHs and HAC materials, are also clearly observed in the spectrum of the heated HACs. These bands agree with those of aromatic bands observed in astronomical observations.

Conclusions. Aromatization of HACs could be a pre-stage in the decomposition process of hydrocarbons that form PAH-clusters in such hot interstellar medium.
\end{abstract}

Key words. astrochemistry - shock waves - methods: laboratory - dust, extinction - infrared: ISM - ISM: supernova remnants

\section{Introduction}

In the interstellar medium (ISM), dust grains suffer several destructive processes that are depending on the shock-velocity as described in Jones et al. (1994, 1996). These authors found that about $80 \%$ of the mass of grains is shattered into very small grains. With shock-velocities between 50 to $200 \mathrm{~km} \mathrm{~s}^{-1}$, about $5-15 \%$ of the initial mass of the grains (radii $\geq 50 \AA$ ) may be converted into small fragments $(\leqslant 14 \AA)$. These fragments presumably have the dimensions of polycyclic aromatic hydrocarbon (PAH) molecules or clusters. Thus, in the ISM, carbonaceous grains may be a source of PAHs, PAH clusters, and small grains. One candidate of carbonaceous dust grains in the ISM is the hydrogenated amorphous carbon (HAC), which is considered as a laboratory analog of interstellar carbonaceous dust (e.g., Colangeli et al. 1997; Schnaiter et al. 1998; Furton et al. 1999; Mennella et al. 1996, 1999; Gadallah et al. 2011, 2012). Recently, comprehensive studies by Jones (2012a,b,c) have demonstrated the structural and optical properties of HACs in detail based on the compositional and spectral modeling of a random covalent network of these materials. Some of these properties are given in detail to explain the evolution of HACs in Sect. 3 .

The earlier studies by Scott \& Duley (1996) and Jones (2009) have shown a progressive evolution of HACs in harsh environments in the ISM. Following these studies, the decomposition products of HACs resulting from the thermal and shock processing in the carbon dust cycle in the ISM may be the most important carriers of the interstellar aromatic emission bands. More precisely, these emission bands are evidence of existing free-flying PAHs, which are expected to be derived from the decomposition of HACs under these harsh processes. In interstellar shocks, the destruction for HACs is more rapid than for graphite (Serra Díaz-Cano \& Jones 2008). Its decomposition, studied experimentally by using low-fluence of a UV (308 nm) laser, may be a source of large PAH and fullerene molecules (Scott et al. 1997).

Within various interstellar regions, variations in the intensity of the aromatic $3.3 \mu \mathrm{m}$ PAH feature and the aliphatic subfeatures in the range of 3.4-3.6 $\mu \mathrm{m}$ were observed according to the local conditions to each region (Sloan et al. 1997; Verstraete et al. 2001; Kondo et al. 2012; Seok et al. 2012). N49 (SNR 0525-66.1) is a detected supernova remnant (SNR), which is interacting with ambient molecular clouds in the Large Magellanic Cloud (LMC). It is a middle-aged SNR, $6600 \mathrm{yr}$ old (Park et al. 2003), with a radiative shock (shock velocity $\approx 100 \mathrm{~km} \mathrm{~s}^{-1}$ ). Seok et al. (2008) estimated the warm dust masses of several SNRs and concluded that the mid-infrared emissions of some SNRs are dominated by thermal emissions from hot dust, that is heated by X-ray emitting plasma. In the study by Otsuka et al. (2010), the dust mass associated with SNR N49 has been estimated to be about $10 M_{\odot}$. Most of the dust in the SNR is probably located in regions with a slow shock, which is needed to produce significant X-ray emission. By assuming amorphous 
carbon as the dust material, Otsuka and collaborators found that the mass of the warm (50-60 K) shock-heated dust component is $0.1 M_{\odot}$ in N49. The shock velocity of N49 is consistent with the velocity range modeled by Micelotta et al. (2010a). In their work, smaller (50 C atoms) and larger (200 C atoms) PAHs have been found to be able to survive below shock velocities of $\approx 100$ and $125 \mathrm{~km} \mathrm{~s}^{-1}$, respectively. The authors concluded that PAH emission is either originating from outside the shocked region or from internal dense clumps where PAHs were not exposed to completely destructive processes in the shocked gas. In other models reported in Bocchio et al. (2012) and Micelotta et al. (2010b), PAHs, depending on their size, are very rapidly destroyed in a hot coronal-type gas and a hot shock gas, respectively. The latter suggests that PAH emission is only possible from dense clumps that are not yet fully heated. Some colder and slow-shock regions with less destructive conditions for PAHs are very likely relevant to the middle-aged SNR N49 (Seok et al. 2012). This assumption agrees with the results of the astronomical observations of the shocked SNR N49 (Seok et al. 2012) and of the galactic superwind of M 82 (Yamagishi et al. 2012). In these objects PAHs could be surviving in protected regions, whereas the presence of these PAHs is mainly due to the fragmentation of larger carbon grains such as HACs.

In the ISM, HACs are evolutionary subjected to various processes such as UV irradiation (Gadallah et al. 2011, 2012; Mennella et al. 1996) and thermal heating (Goto et al. 2003). In addition to the change in the morphology or in the size of dust grains because of sputtering, collision, or erosion mechanisms, the thermal processing (or heating) can physically change the internal structure of HACs, i.e., the atomic bonding and the electronic hybridization. As a result of the decomposition of the HAC's structure, much more aromatic carbon structures can be produced. The structure of HACs is spectroscopically characterized by the aliphatic $\mathrm{C}-\mathrm{H}$ stretching band at $3.4 \mu \mathrm{m}$ and by the aliphatic $\mathrm{C}-\mathrm{H}$ bending bands at 6.85 and $7.25 \mu \mathrm{m}$. The hydrogen-carbon bonds are easily destroyed in a hightemperature shocked medium, resulting in a removal of many of the hydrogen atoms. Consequently, a rearrangement (lattice) of the carbon-carbon bonding and the remaining hydrogen-carbon bonds takes place, leading to the dominant aromatic $\mathrm{sp}^{2}$ hybridization and appearance of the $=\mathrm{C}-\mathrm{H}$ and $\mathrm{C}=\mathrm{C}$ vibrational modes at 3.28 and the $6.2 \mu \mathrm{m}$, respectively.

In this paper, we simulate experimentally the formation of aromatic hydrocarbons in nanosize-scale that occurs because of the fragmentation of HACs in the ISM. The comparison of experimental and observational IR-spectra can demonstrate the existence of aromatic structures in carbonaceous grains within various interstellar regions. In Sect. 2, details of the preparation and processing of HAC and PAH samples are explained, while the results and discussions are given in Sect.3. The astronomical implications are discussed in Sect. 4.

\section{Experiments and Fourier transformation infrared spectroscopy}

\subsection{Sample production}

To emulate various nanoparticles of hydrocarbons in the ISM samples (thin film-like materials in nano-sized scale) were prepared under physical conditions as listed in Table 1. The samples were deposited on $\mathrm{CaF}_{2}$ substrates. Sample \#1 contains a mixture of large PAHs combined with HACs, while \#2, \#3, and \#4 represent HACs, HACs heated up to $703 \mathrm{~K}$, and PAHs, respectively. HAC-samples were produced by the laser ablation method of a rotating graphite target in a quenching gas atmosphere of $\mathrm{He} / \mathrm{H}_{2}(90: 10)$ at a pressure of $4.5 \mathrm{mbar}$. In this method, a Nd:YAG laser with a wavelength of $532 \mathrm{~nm}$ was used; its power was $2.8 \times 10^{10} \mathrm{Wcm}^{-2}$. Using higher values of the laser power, very small quantities of graphite can be evaporated as intense plasma, in which the temperature is higher than $4000 \mathrm{~K}$ (Iida \& Yeung 1994). Collisions between the evaporated carbon components with the quenching gas in a supersonic expansion of the hot plasma cause the condensation of carbon particles. Samples were deposited in a separated chamber at a pressure of $(5 \pm 3) \times 10^{-6}$ Torr (see Fig. 1 in Jäger et al. 2008 for details).

The PAH-samples were prepared by the laser-induced pyrolysis of a gas-phase hydrocarbon (ethylene, $\mathrm{C}_{2} \mathrm{H}_{4}$ ) precursor using a high-power continuous-wave (cw) $\mathrm{CO}_{2}$ laser. The experimental setup was explained in Morjan et al. (2003) and Jäger et al. (2006, 2009). In the current work, the experiment ran with $60 \mathrm{~W}$ radiation of a $\mathrm{cw} \mathrm{CO}_{2}$ laser at a wavelength of $10.6 \mu \mathrm{m}$ with gas flows of $\mathrm{Ar}$ and $\mathrm{C}_{2} \mathrm{H}_{4}$ of $1500 \mathrm{sccm}$ (standard cubic centimeters per minute) and $40 \mathrm{sccm}$, respectively, at a pressure of 750 mbar. The laser power density was approximately $480 \mathrm{Wcm}^{-2}$ for a laser beam of $4 \mathrm{~mm}$ diameter. The gas-phase precursor, $\mathrm{C}_{2} \mathrm{H}_{4}$, absorbed the $\mathrm{CO}_{2}$ laser radiation. Molecules and particles of PAHs were formed in the hot $\left(1100{ }^{\circ} \mathrm{C}\right)$ flame-like condensation zone. Afterward, very small PAHs were removed by methanol extraction, while other PAHs were solved in dichloromethane. Most of the given PAHs in the sample are composed of about 14 to $32 \mathrm{C}$ atoms. The technical details for the production and the spectroscopic extraction are described in Jäger et al. (2006, 2009) and Steglich et al. (2010, 2012).

An HAC sample was heated to simulate experimentally the aromatic emissions produced by decomposition of larger carbon dust grains in SNR N49. Its spectral result was compared with those of a PAH sample. A mixture of HACs and PAHs was also taken into account, in which a thin layer of PAH sample has been covered by another layer of HAC materials. This simple collection of samples may be an analog of hydrocarbon dust grains, which contain aromatic units in a hot ISM.

\subsection{Thermal processing}

Heating is an expected mechanism leading to the decomposition of HAC materials, once they flow in a hot medium such as the interstellar shock region. Therefore, the aromatization by the thermal processing of HACs could be experimentally helpful to understanding the modification of hydrocarbons in a hot medium. In this experiment, the sample \#2 was heated to $703 \mathrm{~K}$ by an electrical heating system in the same chamber as the deposition in high vacuum (approximately $10^{-6}$ Torr). After heating, it was considered as sample \#3. A thermocouple connected with the sample's holder inside the substrate chamber was used to measure the temperature of the sample. In this system, the temperature was controlled to rise slowly to $323 \mathrm{~K}$ and remain constant for at least one hour to remove chemical adsorbents by pumping. The temperature was also kept constant at $473 \mathrm{~K}$ for two hours or more to purge some radicals of hydrocarbons, which were present inside the chamber due to this heating.

\subsection{Infrared measurements}

The Fourier transformation infrared (FTIR) spectroscopy can be used to study the chemical bonding of hydrogen and its concentration in hydrocarbons such as HACs and PAHs. 

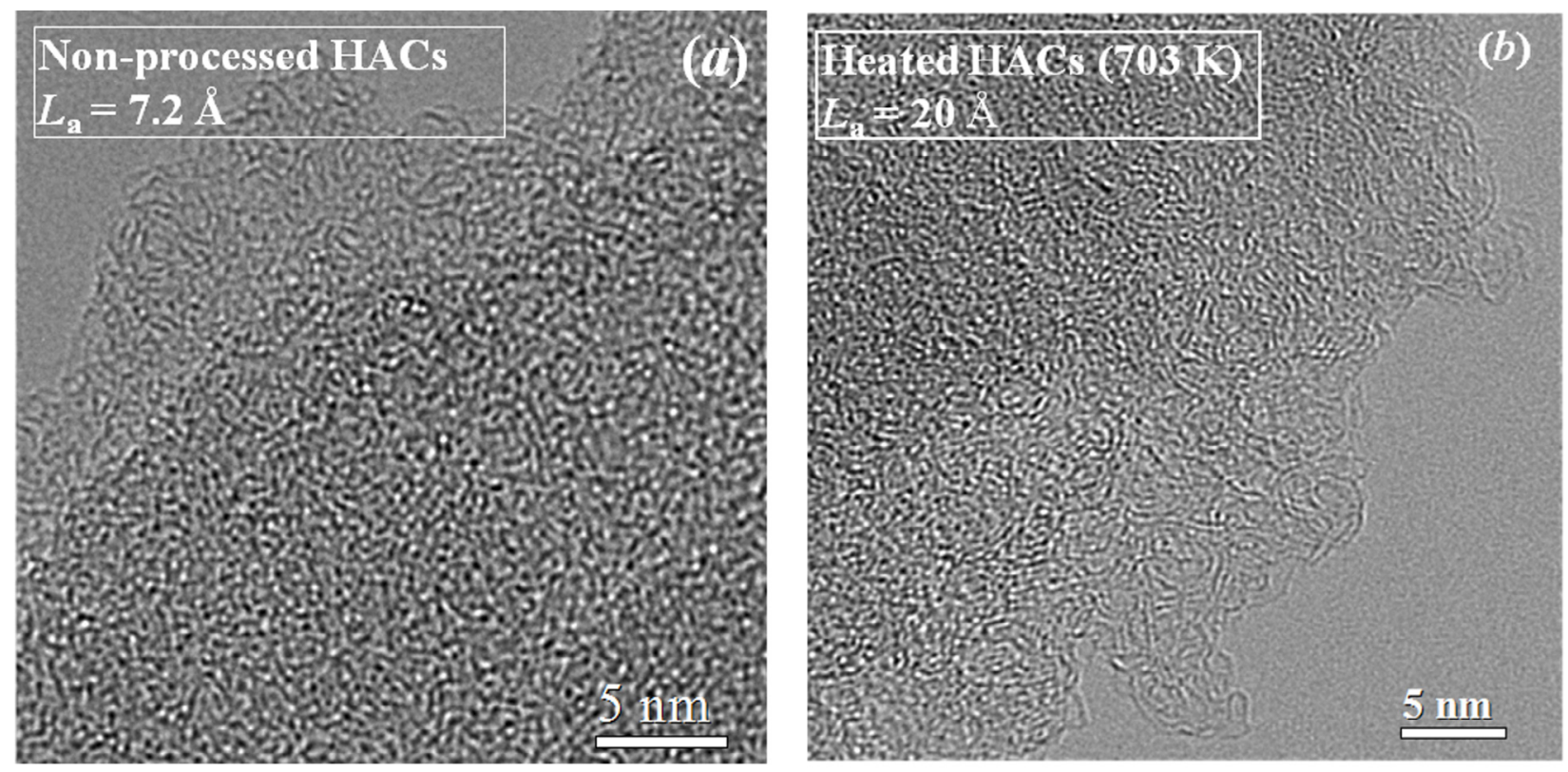

Fig. 1. HRTEM images for non-processed HACs (a) and for heated HACs (b).

Experimentally, vibrational modes of HAC materials (or carbon soot) have been tabulated by several authors (e.g., Dischler et al. 1983; Jäger et al. 2008). In the current study, the IR transmission measurements were taken in situ (ex situ for \#4) by using the FTIR spectrometer Bruker 113v. They were corrected directly during the measurements by using a blank substrate as reference for the transmission spectrum for each sample. A baseline correction of all spectra was performed to subtract the continuum absorption.

\section{Results and discussions}

\subsection{Aromatization of HACs}

According to the structure of HACs, aromatization can be explained through the variation in the average length of the graphene layers $\left(L_{\mathrm{a}}\right)$ with heating. Thus the amorphous structure of the carbonaceous materials can be thermally modified, resulting in more aromatic structures. The linear dimensions of the disordered graphene layers can be measured analytically by high-resolution transmission electron microscopy (TEM) of an HAC material. This material produced by laser ablation is characterized by its bent graphene layers. It is clearly visible in TEM images, as shown in Fig. 1, that most of these layers are strongly deformed with heating. In these TEM images, an increase in $L_{\mathrm{a}}$ of these layers was observed with heating as evidence of forming more aromatic units in the internal structure of HACs. $L_{\mathrm{a}}$ increases from $7.2 \AA$ for non-processed HACs to $20 \AA$ with heating to $703 \mathrm{~K}$, as shown in Fig. 1.

The aromatic structure ( $\mathrm{sp}^{2}$ clustering) is generally given in forms of carbon chains and deformed (five- / six-fold rings) and ordered (six-fold rings) aromatic layers. Both pentagons and hexagons could be particularly important to form small fullerene-like carbon structures. For simplicity, we illustrate the aromatization in amorphous hydrocarbons with aromatic units consisting of six-fold rings. For a single graphene sheet with dimensions of $L_{\mathrm{a}}$, the number of six-fold rings, $N_{\mathrm{R}}$, can be estimated by the product of the integer values $m_{\text {zig }}$ and $n_{\text {arm }}$, which are the number of carbon rings in zigzag and armchair directions, respectively, as shown in Fig. 2a. These numbers can be derived from $L_{\mathrm{a}}$ by using the geometric characterizations of the hexagon form of a carbon ring (with a C-C bond $=1.42 \AA$ );

$m_{\mathrm{zig}}=\frac{L_{\mathrm{a}}-1.23}{2.46}$,
$n_{\mathrm{arm}}=\frac{L_{\mathrm{a}}-0.71}{2.13}$.

Figure $2 \mathrm{~b}$ represents the aromatic domain with $L_{\mathrm{a}}=7.2 \AA$ in the structure of non-processed HACs while Fig. 2c represents that with $L_{\mathrm{a}}=20 \AA$ in the structure of heated HACs. As a result of this concept, we have found that $N_{\mathrm{R}}$ is directly proportional to the temperature of the heating process. It increases from six carbon rings for the non-processed sample to 63 carbon rings for the sample heated up to $703 \mathrm{~K}$. Consequently, this increase is generated by the reduction of the hydrogen fraction, $X_{\mathrm{H}}$, which is a function of the $\mathrm{H} / \mathrm{C}$ ratio $\left(X_{\mathrm{H}}=\mathrm{H} / \mathrm{C} /(1+\mathrm{H} / \mathrm{C})\right)$. This fraction can be estimated from infrared spectroscopy, as will be described in Sect. 3.2. As will be shown there, $X_{\mathrm{H}}$ decreases from 0.44 to about 0.1 in the atomic structure of HACs with heating. In Jones (2012a), the general trends of the fractional abundances of aromatic $\mathrm{sp}^{2} \mathrm{C}=\mathrm{C}$, aromatic and olefinic $\mathrm{sp}^{2}=\mathrm{CH}$ and aliphatic $\mathrm{sp}^{3}$ $\mathrm{CH}$ groups have been shown in dependence on $X_{\mathrm{H}}$ for the extended random covalent network model. Our experimental results of the variation of $L_{\mathrm{a}}$ and $N_{\mathrm{R}}$ with hydrogen loss, due to the thermal processing of HACs, agree with the calculations of those reported in Jones (2012a), in which $L_{\mathrm{a}}$ and $N_{\mathrm{R}}$ for this model vary inversely with $X_{\mathrm{H}}$.

For our original HAC samples with an average $L_{\mathrm{a}}$ of $7.2 \AA$, less than $50 \%$ of the graphene layers may have dimensions like those of compact (circular) PAHs, which need at least dimensions of $8.61 \times 7.1 \AA$ for zigzag and armchair directions, respectively. Others (more than 50\%) may have dimensions like those of catacondensed PAHs, having more open-PAH structures. For the larger dimensions of graphene sheets after heating, similar PAH structures can be mostly compact. In aromatic units with dimensions $L_{\mathrm{a}}=20 \AA$, approximately 63 carbon rings can be arranged to form larger PAHs. 
Table 1. Experimental details for hydrocarbon samples.

\begin{tabular}{|c|c|c|c|c|c|}
\hline Sample & Evaporation method $^{(1)}$ & $\begin{array}{c}\text { Laser power } \\
\left(\mathrm{Wcm}^{-2}\right)\end{array}$ & Atmosphere & $\begin{array}{l}\text { Pressure } \\
\text { (mbar) }\end{array}$ & Product $^{(2)}$ \\
\hline \multirow{2}{*}{$\# 1$} & laser ablation (for HACs) & $2.8 \times 10^{10}$ & He:H (90:10) & 4.5 & \multirow{2}{*}{$\mathrm{PAH}+\mathrm{HAC}$} \\
\hline & laser-induced pyrolysis (for PAHs) & 480 & Ar: $\mathrm{C}_{2} \mathrm{H}_{4}(1500: 40)$ & 750 & \\
\hline$\# 2$ & \multirow{2}{*}{ laser ablation } & \multirow{2}{*}{$2.8 \times 10^{10}$} & \multirow{2}{*}{ He:H (90:10) } & \multirow{2}{*}{4.5} & HAC \\
\hline$\# 3^{(3)}$ & & & & & HAC $(703 \mathrm{~K})$ \\
\hline \#4 & laser-induced pyrolysis & 480 & Ar: $\mathrm{C}_{2} \mathrm{H}_{4}(1500: 40)$ & 750 & PAH \\
\hline
\end{tabular}

Notes. For the laser ablation method, the second harmonic of a pulsed Nd:YAG laser (at $532 \mathrm{~nm}$ ) was used. Samples were produced in a deposition chamber at $(5 \pm 3) \times 10^{-6}$ Torr (Jäger et al. 2008; Gadallah et al. 2011). For the laser-induced pyrolysis method, a high-power continuous-wave (cw) $\mathrm{CO}_{2}$ laser was used with $60 \mathrm{~W}$ radiation power at a wavelength of $10.6 \mu \mathrm{m}$ (Jäger et al. 2006, 2009; Steglich et al. 2010, 2012). Samples were deposited on $\mathrm{CaF}_{2}$ substrates. Sample \#3 was heated to $703 \mathrm{~K}$.

(a)

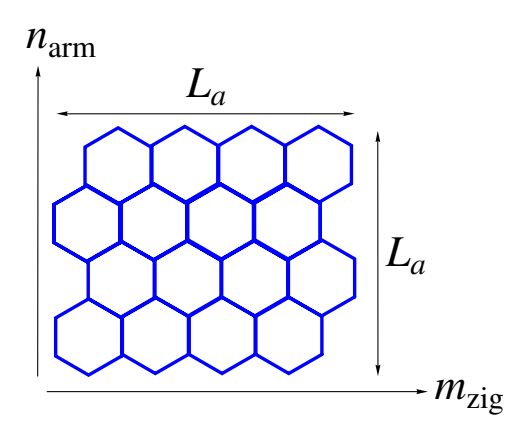

(b)

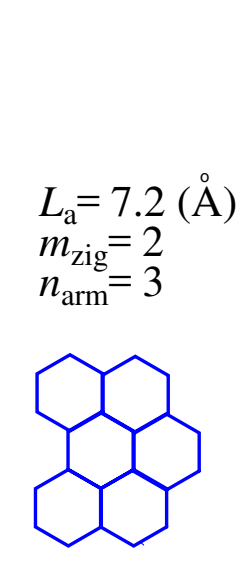

(c)

$$
\begin{aligned}
& L_{\mathrm{a}}=20(\AA) \\
& m_{\mathrm{zig}}=7 \\
& n_{\mathrm{arm}}=9
\end{aligned}
$$

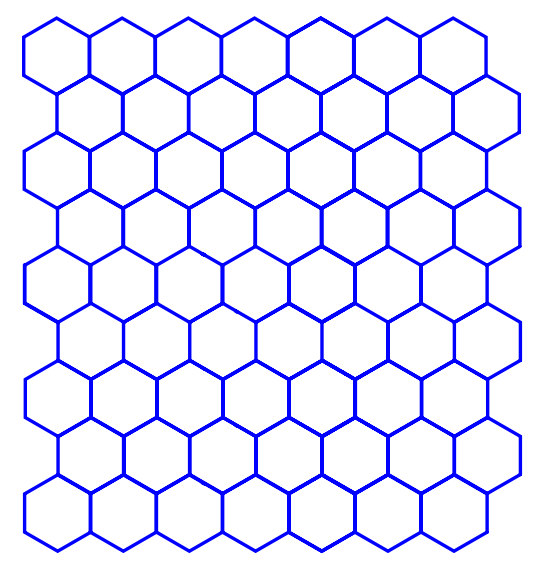

Fig. 2. Structure of aromatic domain $\left(m_{\mathrm{zig}} \times n_{\mathrm{arm}}\right)$ implying six-fold rings, $N_{\mathrm{R}}$, per aromatic cluster in HACs. A graphene sheet with $L_{\mathrm{a}}$ dimensions (a), with $L_{\mathrm{a}}=7.2 \AA$ for non-processed HACs (b), and with $L_{\mathrm{a}}=20 \AA$ for heated (703 K) HACs (c).

\subsection{Mid-IR spectroscopic results}

In this section, experimental mid-infrared absorption spectra of carbonaceous samples are given to show that these hydrocarbons could be analogs of dust grains in interstellar shocked regions. Two aromatic bands at $3.3 \mu \mathrm{m}$ and $6.2 \mu \mathrm{m}$ will be particularly considered for this purpose. Figure 3 shows the spectra of samples \#1, \#2, \#3, and \#4 (compare Table 1) from bottom to top.

In Fig. 3 (left), the spectra in the range from $2.9 \mu \mathrm{m}$ to $3.8 \mu \mathrm{m}$ involve the absorption bands of the alkyne $\mathrm{sp}^{1} \equiv \mathrm{C}-\mathrm{H}$ stretching at $3.03 \mu \mathrm{m}$, the aromatic $\mathrm{sp}^{2}=\mathrm{C}-\mathrm{H}$ stretching at $3.3 \mu \mathrm{m}$, and the aliphatic sp 3 C-H stretching of the $\mathrm{CH}_{n=2,3}$ groups at $3.4 \mu \mathrm{m}$. These bands appear clearly in the spectra of samples \#1 and \#2. In spectra of samples \#3 and \#4, the $3.03 \mu \mathrm{m}$ band completely disappears while the $3.4 \mu \mathrm{m}$ band is very weak compared with that in the other spectra. The $3.3 \mu \mathrm{m}$ band is observed in the spectra of all samples. Its position is slightly shifted toward shorter wavelengths (see also Table 2) and this shift may be attributed to the bending of the graphene layers. The width of this band seems to be slightly broadened because of the likely presence of some olefinic $\mathrm{sp}^{2} \mathrm{CH}_{2}$ and $\mathrm{CH}$ modes at 3.25 and $3.32 \mu \mathrm{m}$, respectively, in particular in the original HAC sample \#2. As shown from the spectra of samples \#1 and \#2, the strength of this band is related to the existence of aromatic clusters in each sample. Obviously, this band is very strong in the spectra of samples \#1 and \#4, which mainly contain PAH-structures. For heated HAC (\#3), which very likely contains PAH-like structures after heating, it becomes also very strong compared with the spectrum of the original HAC sample (\#2). The decomposition of HACs by the thermal processing is indicated by the reduction of the aliphatic $\mathrm{CH}_{n=2,3}$ groups (3.4 $\mu \mathrm{m}$ stretching band). For the original HAC material (\#2), the estimated hydrogen-to-carbon ratio $(\mathrm{H} / \mathrm{C})$ is 0.79 , while for $\# 3(703 \mathrm{~K})$ this fraction is reduced to 0.1 . These values were directly determined based on the prescribed method of the analysis of the $\mathrm{C}-\mathrm{H}$ stretch absorption feature by Jacob \& Unger (1996). More details for the derivation of this fraction are given in Mennella et al. (2002). The corresponding values of $X_{\mathrm{H}}$, which are 0.44 and 0.1 for samples \#2 and \#3 $(703 \mathrm{~K})$, respectively, can be calculated from the $\mathrm{H} / \mathrm{C}$ ratio as described in Gadallah et al. (2011). 


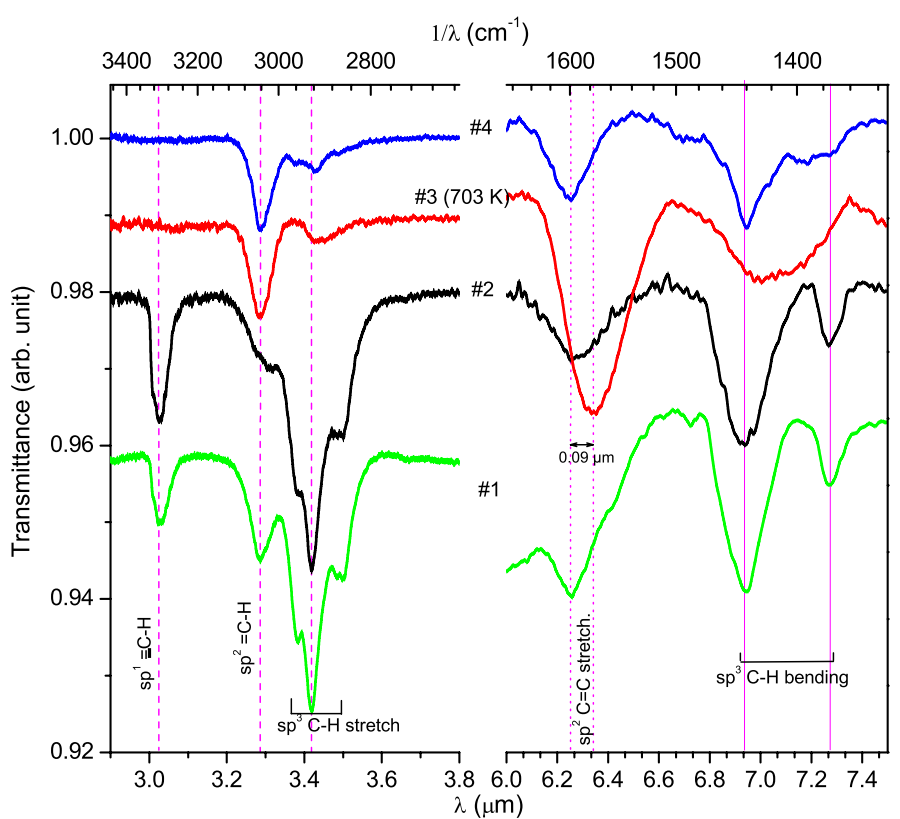

Fig. 3. Experimental spectra in the ranges from $2.9 \mu \mathrm{m}$ to $3.8 \mu \mathrm{m}$ and from $6.0 \mu \mathrm{m}$ to $7.5 \mu \mathrm{m}$ for hydrocarbon samples (\#1 to \#4). The vertical dashed lines at 3.03, 3.3 and $3.4 \mu \mathrm{m}$ bands represent the $\mathrm{sp}^{1} \equiv \mathrm{C}-\mathrm{H}$ stretching, the aromatic $\mathrm{sp}^{2}=\mathrm{C}-\mathrm{H}$ stretching and the aliphatic $\mathrm{sp}^{3}-\mathrm{C}-\mathrm{H}$ stretching, respectively. The vertical dotted lines show the difference in the $6.2 \mu \mathrm{m}$ positions. The vertical solid lines represent the aliphatic $\mathrm{sp}^{3}-\mathrm{C}-\mathrm{H}$ bending at $6.94 \mu \mathrm{m}$ and $7.27 \mu \mathrm{m}$.

The spectra in the $6.0-7.5 \mu \mathrm{m}$ range in Fig. 3 (right) cover the absorption band of the aromatic $\mathrm{sp}^{2} \mathrm{C}=\mathrm{C}$ stretching at $6.2 \mu \mathrm{m}$ and absorption bands of the aliphatic $\mathrm{sp}^{3} \mathrm{C}-\mathrm{H}$ bending at both $6.93 \mu \mathrm{m}$ and $7.27 \mu \mathrm{m}$. The position of the $6.2 \mu \mathrm{m}$ band is almost fixed at $6.25 \mu \mathrm{m}$ for all samples except for that of the heated HAC (\#3), whose spectrum shows that the band is shifted $(\Delta \lambda=0.09 \mu \mathrm{m})$ toward longer wavelengths at approximately $6.35 \mu \mathrm{m}$ (C-class PAHs). This shift is attributed to the reduction of the $\mathrm{C}-\mathrm{H}$ bonds (dehydrogenation) with heating, while the electronic gap energy of HACs decreases with heating, as explained in Gadallah (2010). Where the gap energy decreases approximately from $1.65 \mathrm{eV}$ to $1 \mathrm{eV}$ with heating up to $600{ }^{\circ} \mathrm{C}$. In general, this gap energy declines as a function of the dimensions of the graphene layers and consequently as a function of the number of their rings according to the model of Robertson (1986). As a result of the hydrogen loss, an enhancement in the size of the aromatic clusters ( $\pi$-bonded system of carbon atoms) occurs, which is indicated by the decrease of the gap energy. The big sizes of aromatic units support the formation of colonies of PAH-clusters inside heated HACs. Because of both the destruction of the $\mathrm{C}-\mathrm{H}$ bonds in HACs and the removal of the peripheral hydrogen atoms of the PAHs embedded inside the material, the big sizes of the aromatic units are expected. On the other hand, the aliphatic $\mathrm{sp}^{3} \mathrm{C}-\mathrm{H}$ bending (deformation) bands at both $6.93 \mu \mathrm{m}$ and $7.27 \mu \mathrm{m}$ are observed clearly in samples \#1, \#2, and \#4 while in sample \#3, these bands are broad because of the dominant aromatic structures produced by heating.

The spectrum of sample \#1 in Fig. 3 clearly shows the absorption bands of aliphatic and aromatic structures of a material of HACs combined with a mixture of small PAHs. It is somewhat similar to that of sample \#2, with a clear difference in the strength of the $3.3 \mu \mathrm{m}$ band. This similarity supports the theoretical model developed by Jones et al. (1990), that HACs contain PAH clusters. Furthermore, the spectrum of sample \#3 (HACs
Table 2. Extracted Gaussian fitting parameters (position and width) of aromatic bands.

\begin{tabular}{ccccc}
\hline \hline & \multicolumn{2}{c}{$3.3 \mu \mathrm{m}$} & \multicolumn{2}{c}{$6.2 \mu \mathrm{m}$} \\
\hline Sample & $\begin{array}{c}\lambda_{\mathrm{p}} \\
(\mu \mathrm{m})\end{array}$ & $\begin{array}{c}F W H M \\
(\mu \mathrm{m})\end{array}$ & $\begin{array}{c}\lambda_{\mathrm{p}} \\
(\mu \mathrm{m})\end{array}$ & $\begin{array}{c}F W H M \\
(\mu \mathrm{m})\end{array}$ \\
\hline$\# 1$ & 3.286 & 0.057 & 6.264 & 0.134 \\
$\# 2$ & 3.298 & 0.080 & 6.281 & 0.219 \\
$\# 3$ & 3.287 & 0.052 & 6.350 & 0.227 \\
$\# 4$ & 3.290 & 0.047 & 6.248 & 0.151 \\
\hline
\end{tabular}

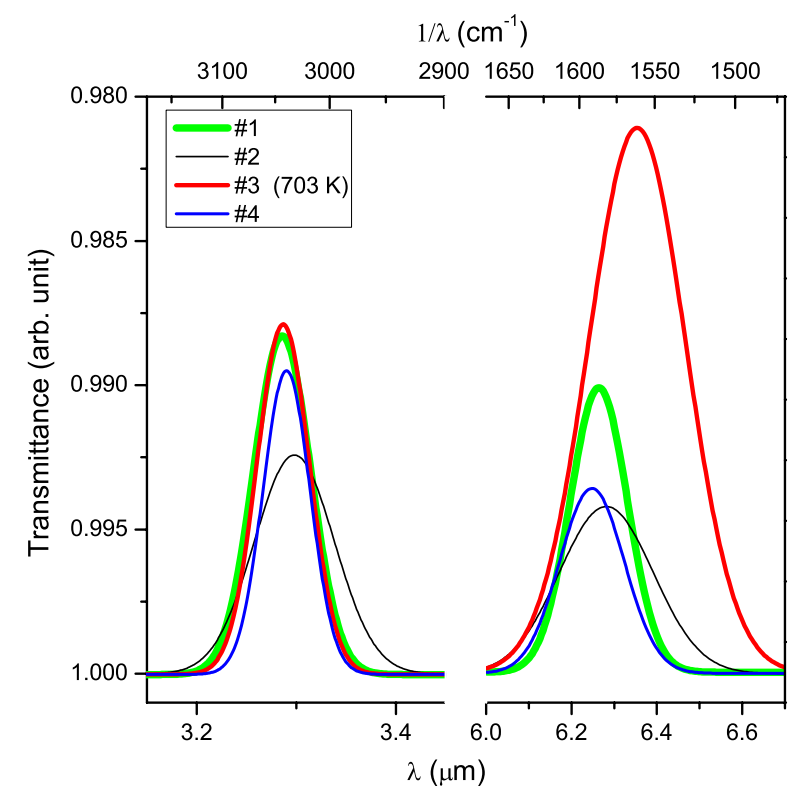

Fig. 4. Gaussian fitting extraction of aromatic bands at $3.3 \mu \mathrm{m}$ and $6.2 \mu \mathrm{m}$ for \#1 to \#4.

heated up to $703 \mathrm{~K}$ ) shows an aromatic band close to $3.3 \mu \mathrm{m}$ that is similar to that in the spectrum of sample \#4 (PAHs). As shown in Fig. 4, Gaussian fitting was used to compare the aromatic bands in all spectra. The wavelength of the band position $\left(\lambda_{\mathrm{p}}\right)$ and the full width at half maximum (FWHM) obtained from the fitting are given in Table 2. For samples \#1, \#3, and \#4, the position of the $3.3 \mu \mathrm{m}$ band is variable only in a range of $\Delta \lambda_{\mathrm{p}}=0.004 \mu \mathrm{m}$, while the FWHM is varying by only $0.01 \mu \mathrm{m}$. The sample \#2 is the only exception where the peak position of this band is located at a longer wavelength than for the others, while the FWHM is considerably larger. This deviation is probably caused by the low contribution of some olefinic $\mathrm{sp}^{2} \mathrm{CH}_{2}$ and $\mathrm{CH}$ modes at 3.25 and $3.32 \mu \mathrm{m}$, respectively, in this sample. The variation in the position of the $6.2 \mu \mathrm{m}$ band for samples \#1 and \#4 (PAHs) is very small $(\Delta \lambda=0.016 \mu \mathrm{m})$ while this band appears at longer wavelengths for samples \#2 (HAC) and \#3 (heated HAC). In parallel, the FWHM of this band for samples \#1 and \#4 is smaller than that for \#2 and \#3.

Figure 5 shows the spectra of our laboratory analogs (HACs) compared with those of the modeled network of HACs in Jones (2012a), in which each spectrum was normalized to its strongest band in that region. In general, these two kinds of the spectral IR-data are consistent in showing the transition from the aliphatic to aromatic structure. This transition is noticed through contrary variations in the intensity of both aromatic and aliphatic bands, which have different trends as a function of $X_{\mathrm{H}}$. On the other hand, this comparison shows a difference between the 


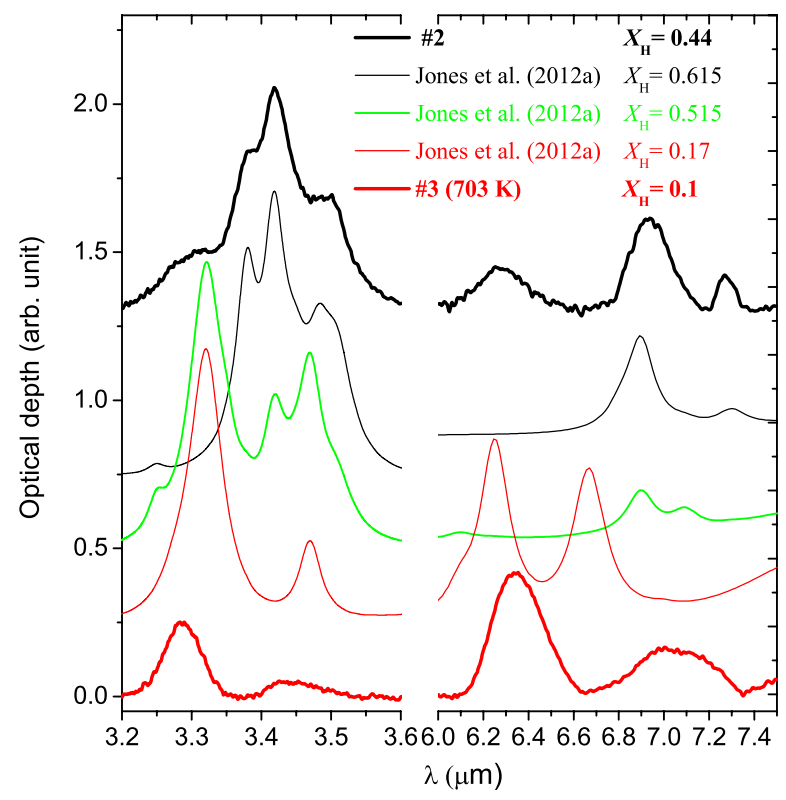

Fig. 5. Mid-IR spectra of HACs as a function in the hydrogen content for our laboratory data (top and bottom spectra) compared with modeled data (three spectra in the middle) in Jones (2012a).

experimental and modeled spectra when the latter are dominated by the $\mathrm{sp}^{2} \mathrm{CH}$ olefinic band at $3.32 \mu \mathrm{m}$ with using lower values of $X_{\mathrm{H}}$, while the experimental results show the aromatic $\mathrm{sp}^{2}$ $\mathrm{CH}$ band (strong) at $3.29 \mu \mathrm{m}$ with a lower value of $X_{\mathrm{H}}$. In another study of the size-dependent model in Jones (2012c), the aromatic $\mathrm{CH}$ stretch at $3.28 \mu \mathrm{m}$ increases, which is attributed to the surface hydrogenation process in $\mathrm{H}$-poor (defective graphite) particles, with smaller subnano sizes. This band is generally consistent with ours and with the $\mathrm{CH}$ emission mode in polycyclic aromatic hydrocarbon (PAH) molecules with peripheral aliphatic structures (Hu \& Duley 2008). There is also an agreement with spectroscopic data of laboratory carbonaceous soot produced under a wide variety of burning conditions (Pino et al. 2008), which shows the aromatic $\mathrm{sp}^{2} \mathrm{CH}$ band at $3.29 \mu \mathrm{m}$ after the transition from aliphatic to aromatic structures. Jones (2012a) has modeled the variation in the structure of HACs with wide variations of $X_{\mathrm{H}}$. He found the transition from aliphatic (dominated) to olefinic structures using $0.5<X_{\mathrm{H}}<0.62$ and from olefinic to aromatic-rich materials using $X_{\mathrm{H}}<0.5$. In the literature, most of the different experimental methods used to produce HAC samples as analogs of carbon dust grains in the ISM (e.g., Dischler et al. 1983; Jacob \& Unger 1996; Furton et al. 1999; Mennella et al. 2002; Gadallah et al. 2011) are limited in producing hydrogen-rich HACs. In these studies, the $\mathrm{H} / \mathrm{C}$ ratio is always lower than 1 yielding $X_{\mathrm{H}}$ lower than 0.5 . In this condition, these analogs can clearly show the aliphatic structures of HACs. But only if the HAC grains are extremely highly hydrogenated with higher $\mathrm{H} / \mathrm{C}$ ratio (Furton et al. 1999), the above model would be able to match the observed optical depth of the interstellar features.

\section{Astronomical implications}

In the $3 \mu \mathrm{m}$ region, we discuss the evolution of the laboratory aromatic and aliphatic (sub-features) $\mathrm{CH}$ stretching at the $3.3 \mu \mathrm{m}$ and around $3.4 \mu \mathrm{m}$ bands, respectively, compared with what is observed in various interstellar environments. The intensities of these bands have been seen in a variable proportion along different interstellar lines of sight.

In both laboratory and astronomical spectra shown in Fig. 6, we can observe a mutual change in the strength of these bands, as illustrated in the spectra in the top and bottom panels. In the top panel, the spectra of objects Sgr A*, GCS 3 and GCS 4 toward the galactic center (Chiar et al. 2000) are dominated by the aliphatic bands referring to the presence of HACs. These spectra agree well with the laboratory spectra of samples \# 1 and \# 2 of HACs. The stretching vibrations of the aliphatic groups are represented by the strong $3.4 \mu \mathrm{m}$ in both the astronomical and experimental spectra. Because the optical depth along the sight line of Sgr A* is higher than those toward GCS 3 and GCS 4, Chiar et al. (2000) found that the Sgr A* spectrum characterizes the molecular cloud dust, while the spectra of both GCS 3 and GCS 4 characterize the dust in the diffuse medium. These aliphatic bands are attributed to the aliphatic $\mathrm{sp}^{3}$ $\mathrm{CH}$ stretching of the $\mathrm{CH}_{n=2,3}$ groups in HACs, which represent the main component of the interstellar dust grains toward the galactic center. The bottom panel in Fig. 6 shows the spectra (two middle spectra; Verstraete et al. 2001) toward the bright interstellar regions (NGC 2023 and the Orion Bar) illuminated directly by stellar radiation. With a very slight variation in its band position, Verstraete et al. (2001) found that the strength of the $3.3 \mu \mathrm{m}$ PAH emission toward the Orion Bar (834 MJy/sr) is much stronger than that toward NGC 2023 (98 MJy/sr). This variation is attributed to the stronger flux and harder radiation field, which heats up these small grains in the Orion Bar. As shown in the same panel (two bottom spectra; Sloan et al. 1997), a similar variation was also observed in the spectra toward two positions located perpendicularly within the transition (Orion Bar emission region) between the ionized Orion Nebula (HII region) to the northwest and the neutral molecular cloud to the southeast. Sloan et al. (1997) concluded that the $3.3 \mu \mathrm{m}$ PAH emission decreases with increasing the depth from the ionized front into neutral region. In this panel, all observed spectra dominated by the $3.3 \mu \mathrm{m}$ PAH band agree well with the laboratory results (two top spectra in this panel) of samples \#3 and \#4. Compared with all spectra with HACs in the top panel, the aliphatic 3.4-3.6 $\mu \mathrm{m}$ subfeatures are much weaker in all spectra in the bottom panel and show notable changes in positions. It is noticeable that the astronomical observations have a similar band at the $3.4 \mu \mathrm{m}$, which may arise from the same origin. This band is shifted toward the longer wavelengths in the spectra of samples \#3 and \#4.

In Kondo et al. (2012), the 3.3 PAH emission and the alphatic 3.4-3.6 $\mu \mathrm{m}$ sub-features were also observed with similar variations in their strength through the region between the nucleus and the starburst ring of the central region of NGC 1097. This study suggested that carbonaceous grains may be shattered in shocks, because of the overflow of the gas into the nucleus in a turbulent motion. Compared with laboratory spectra and those of Kondo et al. (2012), Fig. 7 presents the evolution of these bands in the shocked SNR N49 (Seok et al. 2012). In this shocked region, the aromatic $=\mathrm{CH}$ and $\mathrm{C}=\mathrm{C}$ stretching bands at 3.3 and $6.2 \mu \mathrm{m}$, respectively, are attributed to the presence of small PAHs. The mid-IR spectral data from both AKARI and Spitzer Infrared Spectrograph (IRS) observations were compared with those of the laboratory analogs. To explain this more clearly, it is important to distinguish between PAH emissions from this SNR and those from other back/foreground materials along the line of sight. Therefore, Seok et al. (2012) have taken the SNR N49 located in the LMC. Because it is located away from the galactic disk, the LMC has been considered as 
K. A. K. Gadallah et al.: Analogs of solid nanoparticles as precursors of aromatic hydrocarbons

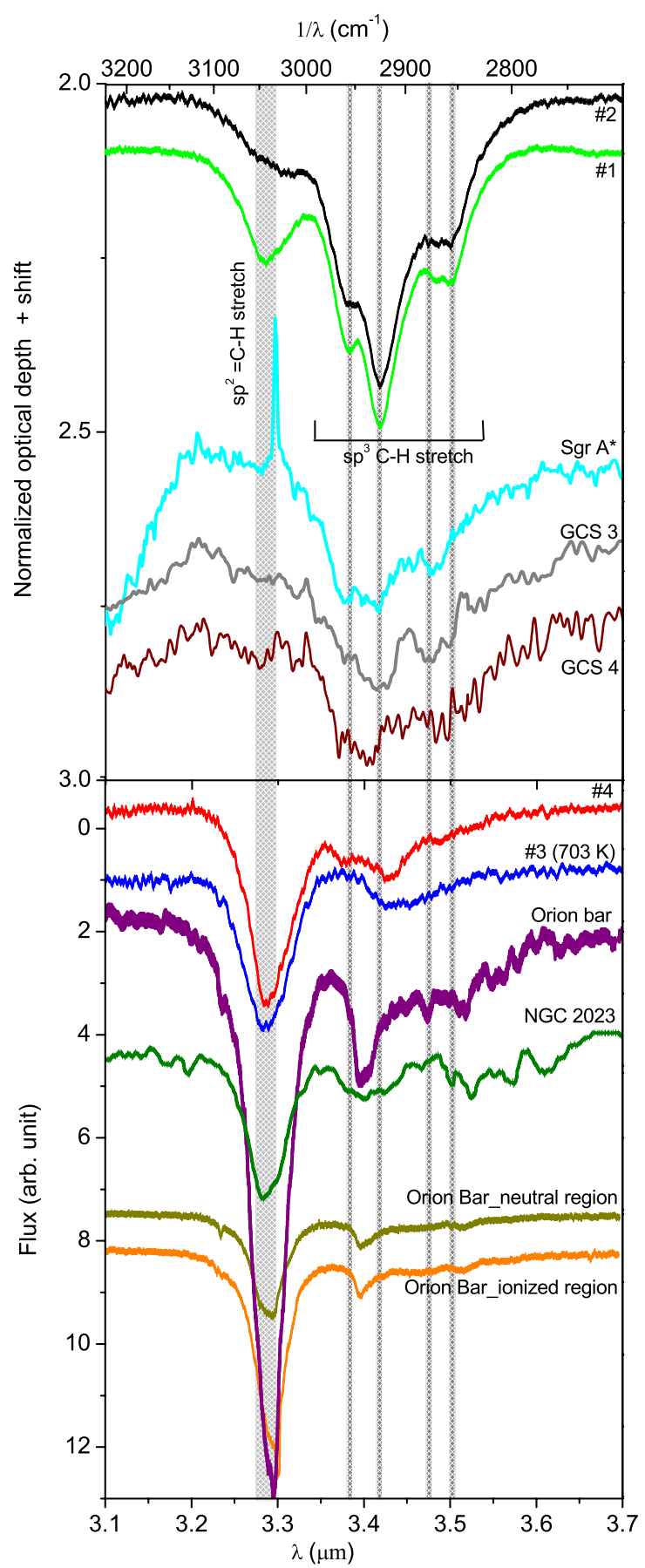

Fig. 6. Compatibility of $3.3 \mu \mathrm{m}$ band (broad gray vertical stripe) and aliphatic $3.4-3.5 \mu \mathrm{m}$ subfeatures (narrow gray vertical stripes) of laboratory results and observations. In the top panel, the spectra of samples \#1 and \#2 are given in addition to those of the ISO-SWS (AOT1) observations toward the galactic center (Sgr A*, GCS 3 and GCS 4; Chiar et al. 2000). In the bottom panel, the spectra of samples \#3 $(703 \mathrm{~K})$ and \#4 are given in addition to those of observations toward the bright interstellar regions (NGC 2023 and the Orion Bar; Verstraete et al. 2001) and of observations toward two positions (ionized and neutral regions) in the Orion Bar emission region (Sloan et al. 1997) from United Kingdom Infrared Telescope (UKIRT).

the best place to observe PAH emissions of SNRs. Moreover, SNR N49 interacts with ambient molecular clouds at the southeast. As shown in the middle panel in Fig. 7, there are AKARI spectra for different regions of SNR N49. The spectra of P1 to P4 were taken for different positions located from east to west and

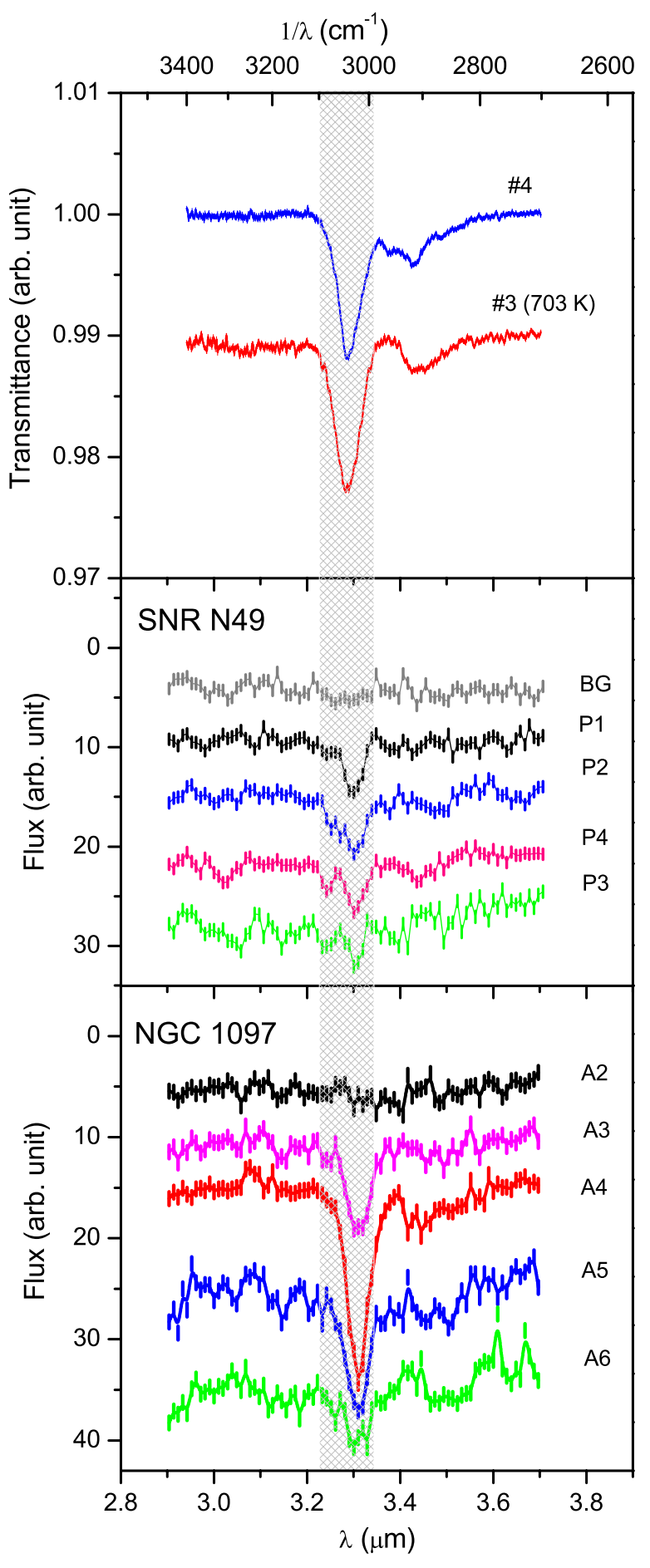

Fig. 7. Compatibility of $3.3 \mu \mathrm{m}$ band (gray vertical stripe) and aliphatic 3.4-3.5 $\mu \mathrm{m}$ subfeatures in laboratory results (top panel) and astronomical-AKARI data for the SNR N49 (middle panel, Seok et al. 2012) and for NGC 1097 (bottom panel, Kondo et al. 2012).

another spectrum of the background (BG) was taken toward a neighboring region to avoid emissions from this remnant. The spectrum of the BG position does not show any emission band at $3.3 \mu \mathrm{m}$, while spectra P1 to P4 clearly show the emission band at $3.3 \mu \mathrm{m}$. Seok et al. (2012) found that the intensity of the $3.3 \mu \mathrm{m}$ feature, which mainly arises from the aromatic $=\mathrm{CH}$ stretching transition, is stronger at P2 in the shock boundary and decreases slightly at P1 (east) outside the shock boundary, indicating the presence of PAHs in the shocked material. On the 


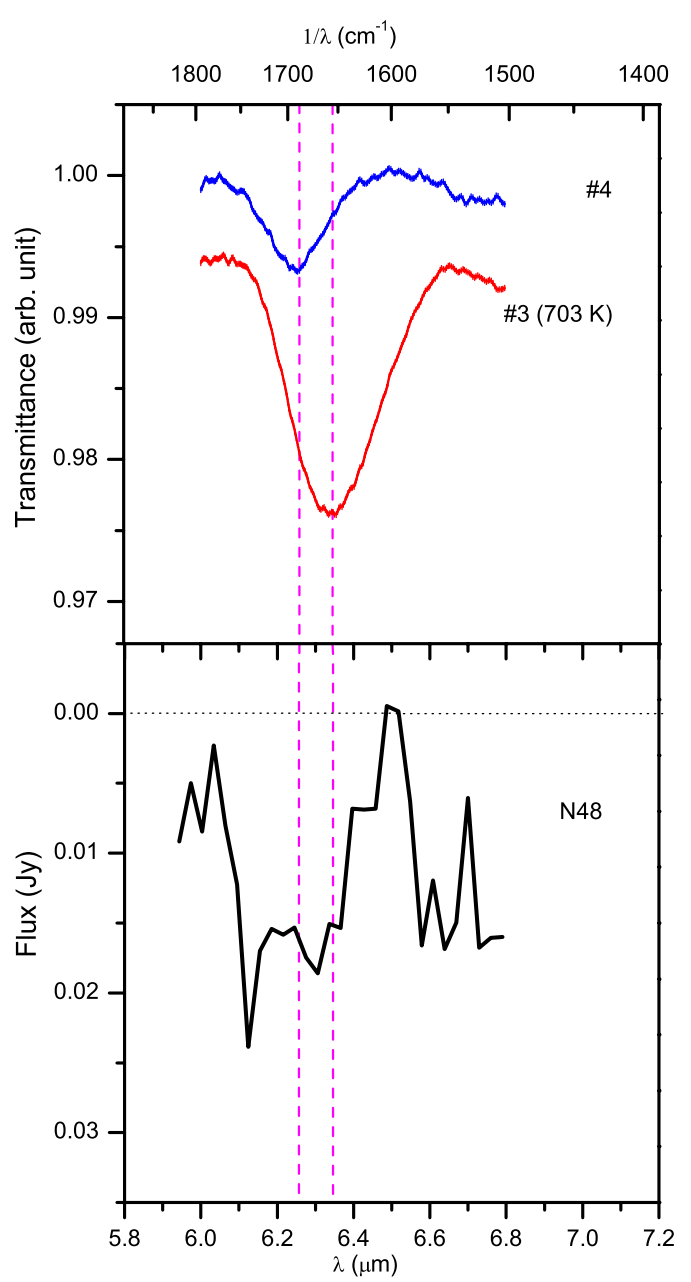

Fig. 8. Compatibility of $6.2 \mu \mathrm{m}$ bands in laboratory results (top panel) and IRS SL spectra (bottom panel) of N49 with Spitzer IRAC spectral response curves. Nod 1 position spectra are used only (Seok et al. 2012). The vertical dashed lines show the band position range at approximately $6.25 \mu \mathrm{m}$ and $6.35 \mu \mathrm{m}$ bands for \#4 and \#3 (703 K).

other side, a remarkable decrease in the intensitycompared with that in P2 is observed in those of P3 and P4 westward passing through the SNR. For NGC 1097, the AKARI/IRC observations were taken in the same way as those of SNR N49. The variation in the intensity of the $3.3 \mu \mathrm{m}$ PAH feature within this SNR is very similar to that through the different positions within the central region of NGC 1097 as shown in the bottom panel in Fig. 7. These positions from A 2 to A6 were chosen from many positions reported in Kondo et al. (2012). Clearly the strong PAH emission peaks at the starburst ring (A4) but decreases toward both the nucleus (A6) and the position (A2) outside the ring. The scenario of the intensity variations of this band coincides in with the view in the size-dependent model by Jones (2012c), in which an increase of the aromatic $3.28 \mu \mathrm{m}$ band is related to the deconstruction of HACs, which could occur in different regions. Compared with our laboratory results, the spectra of \#4 and \#3 (703 K), shown in the top panel in Fig. 7, give an absorption feature close to the $3.3 \mu \mathrm{m}$ band that is similar to those of the aromatic $=\mathrm{CH}$ stretching mode observed in the shocked regions (SNR N49 and NGC 1097). The aromatic structure of hydrocarbons in the SNR $\mathrm{N} 49$ is also supported by the presence of the PAH emission band of the aromatic $\mathrm{C}=\mathrm{C}$ stretching mode at $6.2 \mu \mathrm{m}$ in its Spitzer spectrum (Seok et al. 2012), as shown in the bottom panel of
Fig. 8. The $\mathrm{C}=\mathrm{C}$ stretching is observed in the spectra of samples \#3 (703 K) and \#4 at approximately 6.35 and $6.25 \mu \mathrm{m}$, respectively, as shown in the top panel of this figure.

In van Diedenhoven et al. (2004) at the NASA Ames center for many samples of PAHs with a number of carbon atoms between 10 and 50, the precise position of the $3.3 \mu \mathrm{m}$ is sensitive to the charge state, molecular size, and structure in addition to the molecular heterogeneity of PAHs. Apart from the molecular heterogeneity, we consider the effect of others on this band as follows:

Charge state: for most isolated neutral and anionic PAHs, the band position is found between 3.24 to $3.30 \mu \mathrm{m}$ (in emission, with a redshift, this range is modified to $3.25-3.35 \mu \mathrm{m}$ ), in agreement with the positions of this band for astronomical observations and with those for \#1 and \#4. On the other hand, this range moves toward shorter wavelengths (3.21 to $3.25 \mu \mathrm{m})$ for PAH-cations. As stated in Seok et al. (2012), because PAH features from $\mathrm{C}-\mathrm{H}$ modes at 3.3 and $11.3 \mu \mathrm{m}$ bands are stronger than $\mathrm{PAH}$ features from $\mathrm{C}=\mathrm{C}$ modes at 6.2 and $7.7 \mu \mathrm{m}$ bands, PAHs in SNR N49 are dominantly neutral.

Molecular size: for the PAH size, we found that the interstellar emission at $3.3 \mu \mathrm{m}$ band is is generated by the smallest PAHs while the largest PAHs cause the longer-wavelength PAH bands (Allamandola et al. 1989; Schutte et al. 1993; van Diedenhoven et al. 2004). In agreement with this view, heating of HACs results in the formation of large aromatic units (aromatization). Consequently, the shorter-wavelength PAH band at $3.3 \mu \mathrm{m}$ band has a blueshift while the longer-wavelength PAH band at $6.2 \mu \mathrm{m}$ has a redshift, as listed in Table 2 for samples \#2 and \#3. The $3.3 \mu \mathrm{m}$ band moves from $3.298 \mu \mathrm{m}$ for the original HAC, sample \#2, to shorter wavelengths at $3.287 \mu \mathrm{m}$ for \#3 (703 K) while conversely, the $6.2 \mu \mathrm{m}$ band moves from $6.28 \mu \mathrm{m}$ for \#2 to longer wavelengths at $6.35 \mu \mathrm{m}$ for \#3 (703 K).

Structure: van Diedenhoven et al. (2004) showed that the broadening of the $3.3 \mu \mathrm{m}$ band is related to the irregular PAH edge structure. Straight edges have little interaction between the stretching motions of $\mathrm{CH}$ groups on adjacent rings, and the FWHM is about $0.019 \mu \mathrm{m}$. On the other hand, in case of jagged edges the band is broadened and may reach an FWHM of $0.074 \mu \mathrm{m}$. This range of widths mostly covers those of our current samples between $0.045 \mu \mathrm{m}$ and $0.08 \mu \mathrm{m}$, revealing the dominance of irregular jagged edges. In the spectra of N49 shown in the middle panel of Fig. 7, small PAHs are the dominant contribution for the $3.3 \mu \mathrm{m}$ band, as concluded in Seok et al. (2012). The width of this band is in the range between $0.04 \mu \mathrm{m}$ and $0.05 \mu \mathrm{m}$. It agrees with the typical values for samples \#3 and \#4, which are 0.05 and $0.047 \mu \mathrm{m}$, respectively.

Because of the relatively high ratios of the strength of the $6.2 \mu \mathrm{m}$ and the $3.3 \mu \mathrm{m}$ bands relative to those of the $7.7 \mu \mathrm{m}$ and the $11.3 \mu \mathrm{m}$ bands, Seok and collaborators found that this trend agrees with the formation of small PAHs by fragmentation from larger carbonaceous grains. This is a key point for the discussion of the presence and origin of aromatic hydrocarbons in SNR N49. In general, dust may come from the supernova ejecta or is formed from interstellar materials. In the ISM, aromatic hydrocarbons can be formed after the dehydrogenation process of HACs during the transition from the asymptotic giant branch to proto-planetary nebulae. The change in the aliphatic structure is caused by the environmental changes of increasing stellar temperature, less protective dusty environments, and the outbreak of harsh of UV radiation (Goto et al. 2003, 2007; Kwok et al. 2001). Obviously, as discussed in Seok et al. (2012), the PAH processing in SNR N49 reveals a morphology of this SNR with physically different regions. These include the diffuse inter-cloud 
medium in which the hot gas region with X-ray emission is generated by the fast shock; the dense ambient medium with ionic line emissions that produces the bright optical filaments; and the dense clumps that are affected by slow shocks. With fast shocks ( shock velocity $=300 \mathrm{~km} \mathrm{~s}^{-1}$ ), most PAHs are completely destroyed soon after being swept up by shocks because the lifetime of PAHs, with small or large $\mathrm{C}$ atoms, is not long enough to be detected (Micelotta et al. 2010a). In less destructive environments in a dense medium, small PAHs (50 C atoms) may survive at a shock velocity $<100 \mathrm{~km} \mathrm{~s}^{-1}$, while larger PAHs $(200 \mathrm{C}$ atoms) may survive at a shock velocity $<125 \mathrm{~km} \mathrm{~s}^{-1}$ (Micelotta et al. 2010b). According to Draine \& McKee (1993), PAHs may survive or be formed in shocked environments in dense clumps with a shock velocity $=50 \mathrm{~km} \mathrm{~s}^{-1}$. The theoretical study by Draine \& Li (2007) confirmed this trend, according to which small PAHs with $<100 \mathrm{C}$ atoms can cause the $3.3 \mu \mathrm{m}$ feature. we conclude that PAH emission can be observed when the shock is sufficiently slow, provided the existence of a heating source such as UV photons.

The heating of sample \#3 $(703 \mathrm{~K})$ results in a dramatic reduction in the $\mathrm{C}-\mathrm{H}$ stretching at $3.4 \mu \mathrm{m}$ and its spectrum becomes similar to that of \#4 (see Fig. 7), which contains smaller PAHs between 14 and $32 \mathrm{C}$ atoms. Sample \#3 (703 K) seems to simulate the dehydrogenation of the dust grains (HACs) associated with SNR N49. As shown in the bottom and middle panels in Fig. 7, the weak aliphatic subfeatures around $3.4 \mu \mathrm{m}$ are an interesting features that are observed with slightly different position according to the local conditions to each region. These weak bands are also observed in laboratory spectra of samples \#3 (703 K) and \#4. They are most likely due to the residuals of different subgroups showing the aliphatic $\mathrm{CH}_{n}(n=1-2)$ vibration mode. Finally, the similarity in the spectroscopic properties of the laboratory samples and those of observed spectra provides the chemical composition of aromatic materials in the shocked regions in the ISM.

\section{Conclusions}

We provided experimental results on the aromatic structure of hydrocarbons, such as a mixture of small PAHs, HACs and heated HACs. This structure can be observed in the evolution of graphene layers through aromatization of HACs, with heating and consequently in the aromatic spectral mid-IR bands. In comparison, it seems that the spectroscopic properties of heated HACs are very similar to those of the PAHs. The spectrum of the combination of the HAC and PAH materials may refer to the mixed dust grains in the ISM. The aromatization due to the destructive thermal processing of HACs may be a pre-stage of forming PAH-clusters in carbonaceous grains in a hot ISM. Both the PAHs and heated HACs can simulate the aromatic structure of associated dust grains in the shocked interstellar regions. This structure is confirmed by the absorption bands at $3.3 \mu \mathrm{m}$ and $6.2 \mu \mathrm{m}$ due to the $=\mathrm{CH}$ and $\mathrm{C}=\mathrm{C}$ stretching modes, respectively. These laboratory aromatic materials could represent small PAHs in dust grains, which are associated with the SNR or involved within the SNR due to the interaction with the molecular clumps. In these clumps small PAHs can survive in the slowshocked regions.
Acknowledgements. We are very grateful to the laboratory group at Friedrich Schiller University Jena, Germany, in particular Mathias Steglich, for helping us to prepare PAH samples. We would also like to thank Takashi Onaka from the University of Tokyo, Japan for providing the AKARI and Spitzer IR data for SNR N49 and wish thank Toru Kondo from Nagoya University, Japan for providing the AKARI IR data for NGC 1097. Finally, we gratefully acknowledge the referee Anthony Jones for his valuable comments and suggestions.

\section{References}

Allamandola, L. J., Tielens, A. G. G. M., \& Barker, J. R. 1989, ApJS, 71, 733 Bocchio, M., Micelotta, E. R., Gautier, A.-L., \& Jones, A. P. 2012, A\&A, 545, A124

Chiar, J. E., Tielens, A. G. G. M., Whittet, D. C. B., et al. 2000, ApJ, 537, 749

Colangeli, L., Bussoletti, E., Pestellini, C. C., et al. 1997, Adv. Space Res., 20, 1617

Dischler, B., Bubenzer, A., \& Koidl, P. 1983, Appl. Phys. Lett., 42, 636

Draine, B. T., \& Li, A. 2007, ApJ, 657, 810

Draine, B. T., \& McKee, C. F. 1993, ARA\&A, 31, 373

Furton, D. G., Laiho, J. W., \& Witt, A. N. 1999, ApJ, 526, 752

Gadallah, K. 2010, Ph.D. Thesis, Friedrich-Schiller-University Jena Univ., Diss., 523.1125 [DDC22ger], 520 Astronomie, http://d-nb.info/1010641263

Gadallah, K. A. K., Mutschke, H., \& Jäger, C. 2011, A\&A, 528, A56

Gadallah, K. A. K., Mutschke, H., \& Jäger, C. 2012, A\&A, 544, A107

Goto, M., Gaessler, W., Hayano, Y., et al. 2003, ApJ, 589, 419

Goto, M., Kwok, S., Takami, H., et al. 2007, ApJ, 662, 389

Hu, A., \& Duley, W. W. 2008, ApJ, 677, L153

Iida, Y., \& Yeung, E. S. 1994, Appl. Spectrosc., 48, 945

Jacob, W., \& Unger, M. 1996, Appl.Phys. Lett., 68, 475

Jäger, C., Krasnokutski, S., Staicu, A., et al. 2006, ApJS, 166, 557

Jäger, C., Mutschke, H., Henning, T., \& Huisken, F. 2008, ApJ, 689, 249

Jäger, C., Huisken, F., Mutschke, H., Jansa, I. L., \& Henning, T. 2009, ApJ, 696, 706

Jones, A. P. 2009, in Cosmic Dust - Near and Far, eds. T. Henning, E. Grün, \& J. Steinacker, ASP Conf. Ser., 473, 414

Jones, A. P. 2012a, A\&A, 540, A1

Jones, A. P. 2012b, A\&A, 540, A2

Jones, A. P. 2012c, A\&A, 542, A98

Jones, A. P., Duley, W. W., \& Williams, D. A. 1990, QJRAS, 31, 567

Jones, A. P., Tielens, A. G. G. M., Hollenbach, D. J., \& McKee, C. F. 1994, ApJ, 433, 797

Jones, A. P., Tielens, A. G. G. M., \& Hollenbach, D. J. 1996, ApJ, 469, 740

Kondo, T., Kaneda, H., Oyabu, S., et al. 2012, ApJ, 751, L18

Kwok, S., Volk, K., \& Bernath, P. 2001, ApJ, 554, L87

Mennella, V., Colangeli, L., Palumbo, P., et al. 1996, ApJ, 464, L191

Mennella, V., Brucato, J. R., Colangeli, L., \& Palumbo, P. 1999, ApJ, 524, L71

Mennella, V., Brucato, J. R., Colangeli, L., \& Palumbo, P. 2002, ApJ, 569, 531

Micelotta, E. R., Jones, A. P., \& Tielens, A. G. G. M. 2010a, A\&A, 510, A37

Micelotta, E. R., Jones, A. P., \& Tielens, A. G. G. M. 2010b, A\&A, 510, A36

Morjan, I., Voicu, I., \& Dumitrache, F. 2003, Carbon, 41, 2913

Otsuka, M., van Loon, J. T., Long, K. S., et al. 2010, A\&A, 518, L139

Park, S., Burrows, D. N., Garmire, G. P., et al. 2003, ApJ, 586, 210

Pino, T., Dartois, E., Cao, A., et al. 2008, A\&A, 490, 665

Robertson, J. 1986, Adv. Phys., 35, 317

Schnaiter, M., Mutschke, H., Dorschner, J., Henning, T., \& Salama, F. 1998, ApJ, 498, 486

Schutte, W. A., Tielens, A. G. G. M., \& Allamandola, L. J. 1993, ApJ, 415, 397

Scott, A., \& Duley, W. W. 1996, ApJ, 472, L123

Scott, A., Duley, W. W., \& Pinho, G. P. 1997, ApJ, 489, L193

Seok, J. Y., Koo, B.-C., Onaka, T., et al. 2008, PASJ, 60, 453

Seok, J. Y., Koo, B.-C., \& Onaka, T. 2012, ApJ, 744, 160

Serra Díaz-Cano, L., \& Jones, A. P. 2008, A\&A, 492, 127

Sloan, G. C., Bregman, J. D., Geballe, T. R., Allamandola, L. J., \& Woodward, C. E. 1997, ApJ, 474, 735

Steglich, M., Jäger, C., Rouillé, G., et al. 2010, ApJ, 712, L16

Steglich, M., Carpentier, Y., Jäger, C., et al. 2012, A\&A, 540, A110

van Diedenhoven, B., Peeters, E., Van Kerckhoven, C., et al. 2004, ApJ, 611, 928

Verstraete, L., Pech, C., Moutou, C., et al. 2001, A\&A, 372, 981

Yamagishi, M., Kaneda, H., Ishihara, D., et al. 2012, A\&A, 541, A10 